\title{
CHARMM36 Lipid Force Field with Explicit Treatment of Long-Range Dispersion: Parametrization and Validation for PE, PG, and ether lipids
}

\section{Supporting Information}

\author{
Yalun Yu, ${ }^{\mathrm{a}, \mathrm{b}}$ Andreas Krämer, ${ }^{\mathrm{b}}$ Richard M. Venable, ${ }^{\mathrm{b}}$ Bernard R. Brooks ${ }^{\mathrm{b}}$, \\ Jeffery B. Klauda, *.a,c and Richard W. Pastor*,b
}

aBiophysics Graduate Program and 'Department of Chemical and Biomolecular Engineering, University of Maryland, College Park, Maryland 20742, USA

bLaboratory of Computational Biology, National Heart, Lung and Blood Institute, National Institutes of Health, Bethesda, MD 20892, USA 
Table S1. Comparison of ether linkage partial charges (e) for C36/LJ-PME and C36.

\begin{tabular}{|l|l|l|}
\hline atom & C36 & C36/LJ-PME \\
\hline C2 & 0.13 & 0.1494 \\
\hline HS & 0.01 & 0.0100 \\
\hline O21 & -0.56 & -0.6228 \\
\hline C21 & 0.40 & 0.4142 \\
\hline H1R & 0.01 & 0.0108 \\
\hline H1S & 0.01 & 0.0108 \\
\hline C22 & -0.18 & -0.1556 \\
\hline H2R & 0.09 & 0.0916 \\
\hline H2S & 0.09 & 0.0916 \\
\hline C3 & 0.08 & 0.0706 \\
\hline HX & 0.02 & 0.0344 \\
\hline HY & 0.02 & 0.0344 \\
\hline O31 & -0.56 & -0.6228 \\
\hline C31 & 0.40 & 0.4142 \\
\hline H1X & 0.02 & 0.0208 \\
\hline H1Y & 0.02 & 0.0208 \\
\hline C32 & -0.18 & -0.1556 \\
\hline H2X & 0.09 & 0.0916 \\
\hline H2Y & 0.09 & 0.0916 \\
\hline & & \\
\hline
\end{tabular}


Table S2. Fitting parameters used for the PSD model. $H$ is the average height of water layers above and below the membrane, $L$ is the size of the simulation box in $x / y$, and $\eta_{f}$ is the water viscosity. $H$ and $L$ are averages from simulations.

\begin{tabular}{|c|c|c|c|c|}
\hline system & $H(\AA)$ & $L(\AA)$ & $\eta_{f}(\mathrm{P})$ & $b\left(10^{7} \mathrm{P} / \mathrm{cm}\right)$ \\
\hline DPPC, 72 lipids & 14.3 & 47.5 & $0.00252(323 \mathrm{~K})^{1}$ & 1 \\
\hline DPPC, 288 lipids & 14.3 & 95.0 & 0.00252 & 1 \\
\hline DPPC, 648 lipids & 14.3 & 142.5 & 0.00252 & 1 \\
\hline DOPC, 72 lipids & 14.6 & 50.0 & $0.00299(303 \mathrm{~K})^{1}$ & 1 \\
\hline DOPC, 288 lipids & 13.3 & 100.0 & 0.00299 & 1 \\
\hline DOPC, 648 lipids & 14.6 & 150.0 & 0.00299 & 1 \\
\hline
\end{tabular}



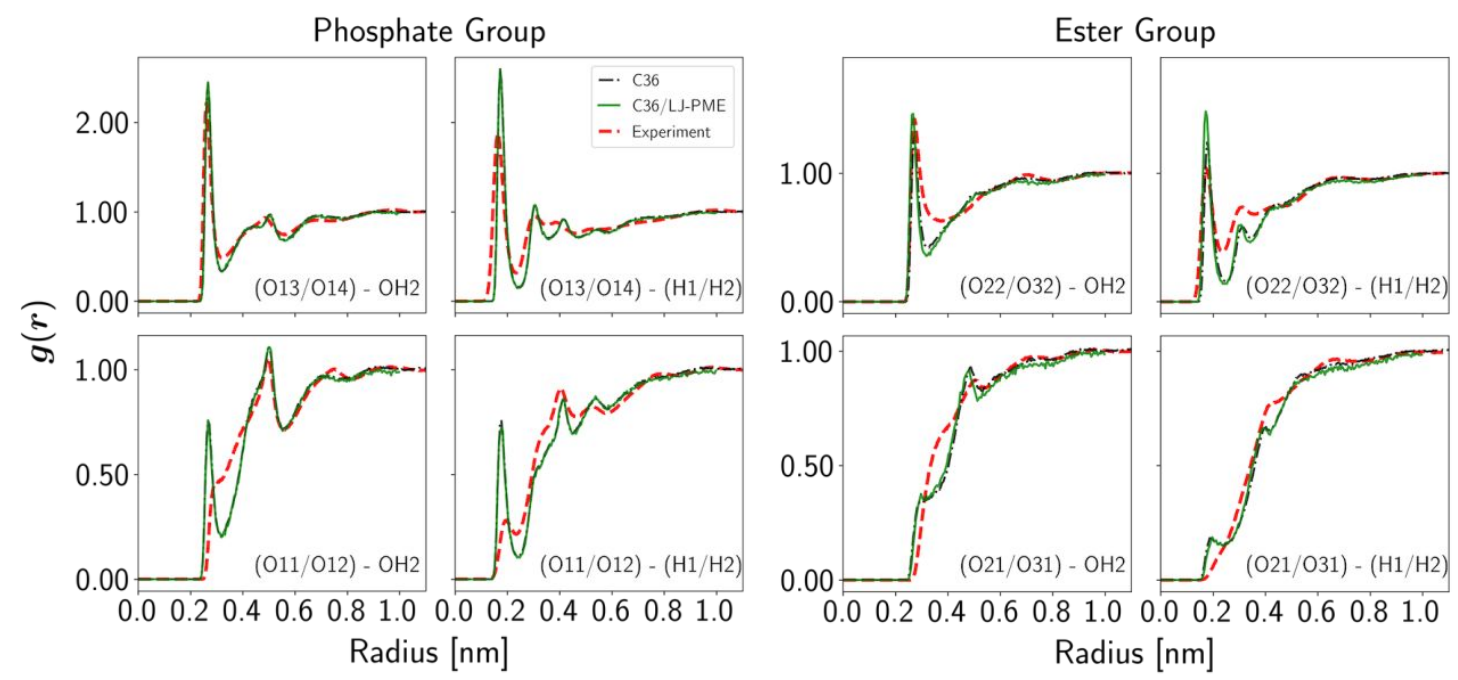

Figure S1. Atom-pair-specific RDFs between water and $\mathrm{C}_{3}-\mathrm{PC}$ in solution for C36/LJ-PME and C36. Experimental data from Foglia et al. ${ }^{2}$

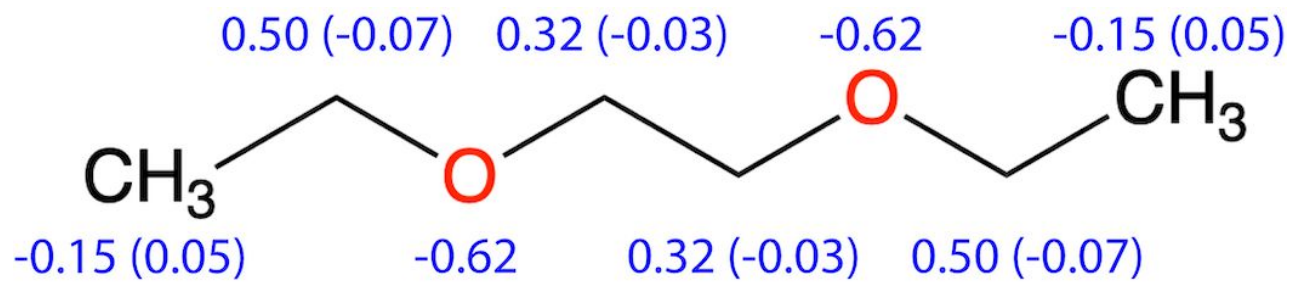

Figure S2. Partial atomic charges of DEOE used for PES. 

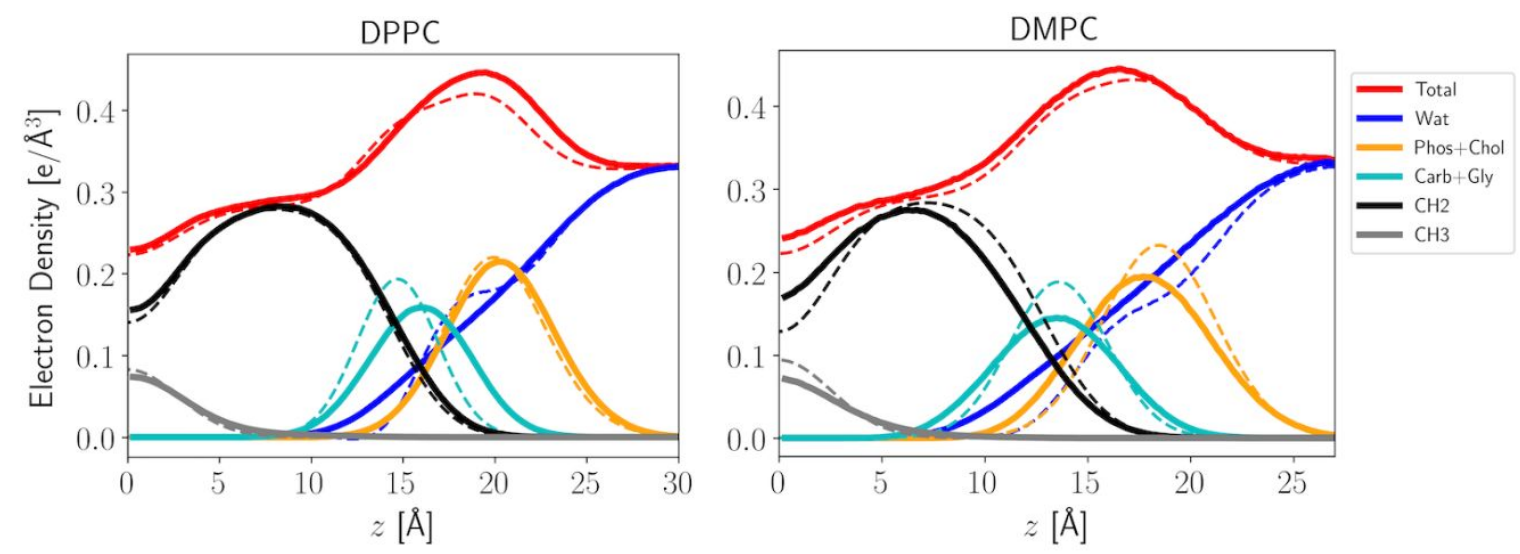

Figure S3. Electron density profiles for DPPC and DMPC bilayers. C36/LJ-PME in solid line and experiment $^{3}$ in dashed line. Wat: water; Phos: phosphate; Chol: choline; Carb: carbonyl; Gly: glycerol; $\mathrm{CH} 2$ : methylene; $\mathrm{CH} 3$ : methyl.

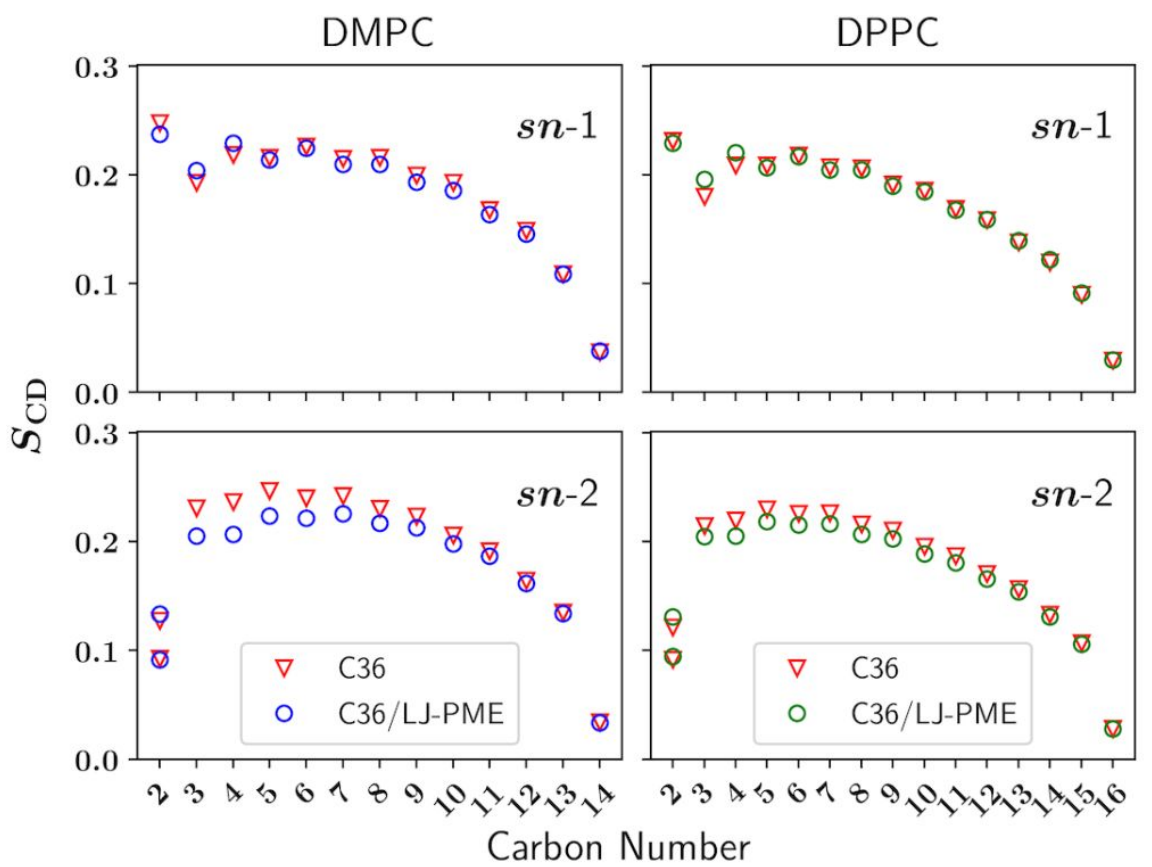

Figure S4. Comparison of chain order parameters between C36/LJ-PME and C36. 


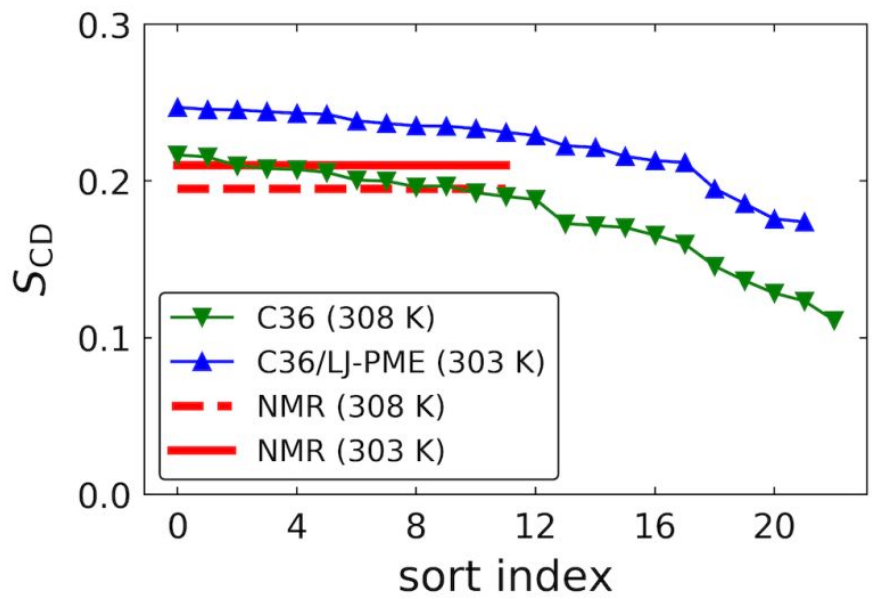

Figure S5. Sorted order parameters from both chains for DMPG bilayer. C36 data from Venable et al. ${ }^{4}$ Experimental data from Loew et al. ${ }^{5}$
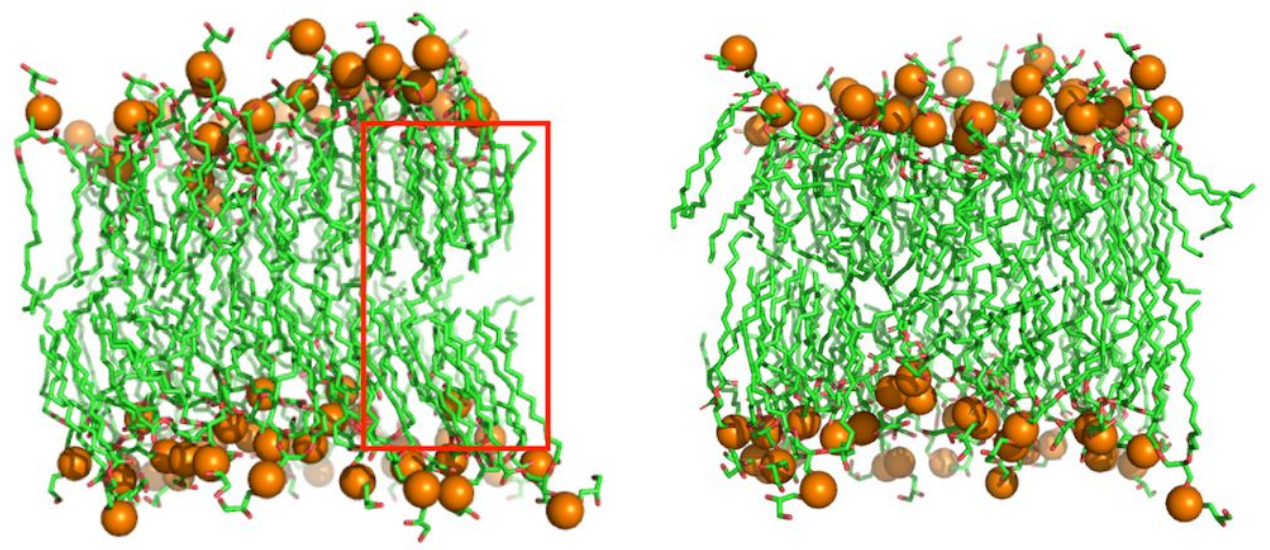

Figure S6. Snapshots from the DMPG bilayer (303.15 K) simulation. Left: snapshot at $120 \mathrm{~ns}$; right: snapshot at $300 \mathrm{~ns}$ (end of simulation). Transient domain containing extended hydrocarbon chains is highlighted in red rectangular. 


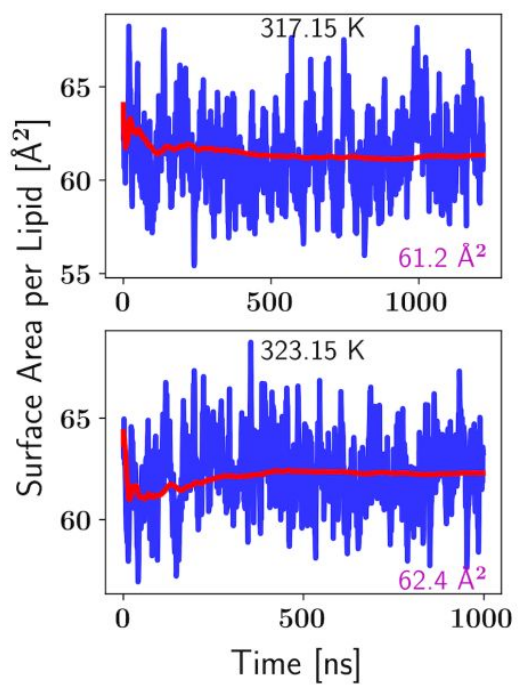

Figure S7. Surface area per lipid of DPPC bilayer at $317.15 \mathrm{~K}$ and $323.15 \mathrm{~K}$ during microsecond simulation using C36/LJ-PME. Average area after 200 ns shown on the bottom right.

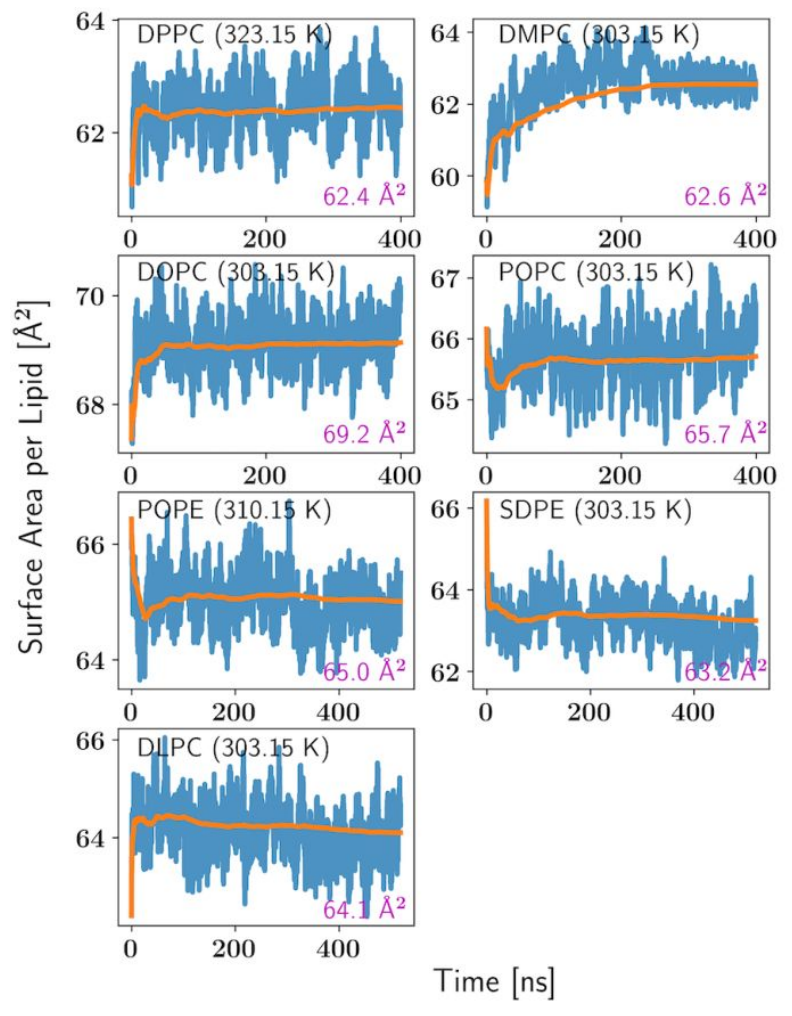

Figure S8. Surface area per lipid for 1152-lipid bilayers. SDPE (PE 18:0,22:6). Average area after 200 ns shown on the bottom right. DPPC, DMPC, DOPC, POPC simulations used parameters from the Global parametrization in Paper I. 


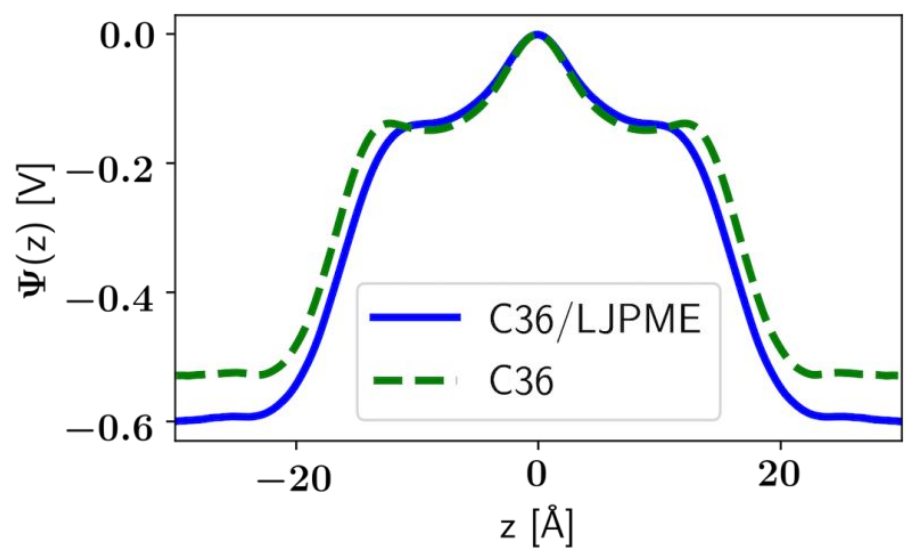

Figure S9. Electrostatic potential drop for DPPC bilayer at $323.15 \mathrm{~K}$.

\section{References:}

1. Venable, R. M.; Hatcher, E.; Guvench, O.; MacKerell, A. D.; Pastor, R. W., Comparing Simulated and Experimental Translation and Rotation Constants: Range of Validity for Viscosity Scaling. J. Phys. Chem. B 2010, 114 (39), 12501-12507.

2. Foglia, F.; Lawrence, M. J.; Lorenz, C. D.; McLain, S. E., On the Hydration of the Phosphocholine Headgroup in Aqueous Solution. J. Chem. Phys. 2010, 133 (14), 145103.

3. Kučerka, N.; Nieh, M. P.; Katsaras, J., Fluid Phase Lipid Areas and Bilayer Thicknesses of Commonly Used Phosphatidylcholines as a Function of Temperature. Biochimica et Biophysica Acta - Biomembranes 2011, 1808 (11), 2761-2771.

4. Venable, R. M.; Luo, Y.; Gawrisch, K.; Roux, B.; Pastor, R. W., Simulations of Anionic Lipid Membranes: Development of Interaction-Specific Ion Parameters and Validation Using NMR Data. J. Phys. Chem. B 2013, 117 (35), 10183-10192.

5. Loew, C.; Riske, K. A.; Lamy, M. T.; Seelig, J., Thermal Phase Behavior of DMPG Bilayers in Aqueous Dispersions as Revealed by 2H- and 31P-NMR. Langmuir 2011, 27 (16), 10041-10049. 